\title{
Estratificação Residencial, Valoração do Trabalho Doméstico e Uso do Tempo: Contribuições para a Análise do Caso do Brasil
}

\author{
Neuma Aguiar ${ }^{1}$ \\ Arnaldo Mont'Alvão ${ }^{2}$ \\ ${ }^{1}$ Universidade Federal de Minas Gerais (UFMG), Belo Horizonte, MG, Brasil. E-mail: \\ neumaaguiar@hotmail.com \\ ${ }^{2}$ Universidade do Estado do Rio de Janeiro (UERJ), Rio de Janeiro, RJ, Brasil. E-mail: \\ almontalvao@gmail.com
}

\section{INTRODUÇÃO}

F ste artigo apresenta a construção de uma escala de estratificação

E residencial (EER) como instrumento de interesse para o estudo das desigualdades em vigor no interior de uma sociedade, possibilitando que se estime a posição social de residentes em um mesmo domicílio numa população determinada. A principal contribuição desta metodologia é atribuir uma posição social aos moradores que não auferem rendimentos, embora possam contribuir ativamente, com trabalho diário, para a manutenção do domicílio, em lugar de considerar apenas aqueles membros do grupamento familiar que geram renda. Essa medida permite observar o efeito das disparidades sociais no uso do tempo pelos membros dos grupos domésticos na população estudada, incluindo, entre outras dimensões, o tempo por eles dedicado ao trabalho domiciliar não remunerado.

A análise da relação entre estratificação residencial e o tempo devotado por homens e mulheres, às atividades domésticas despertou nosso interesse na observação de empreendimentos semelhantes, que procuravam atribuir um valor econômico ao trabalho doméstico não remunerado, com auxílio das pesquisas de uso do tempo. Constatamos, com esse procedimento, que a escala de estratificação residencial possibilita a valoração do trabalho doméstico. Ao contemplar a Economia Doméstica enquanto disciplina, desvendamos alguns de seus pressupostos, derivados das desigualdades sociais existentes na valoração das

DADOS - Revista de Ciências Sociais, Rio de Janeiro, vol. 60, no2, 2017, pp. 331 a 357.

http://dx.doi.org/10.1590/001152582017122 
atividades não pagas, tais como a escolha da ocupação de referência, os bens domiciliares envolvidos na produção doméstica, além de características do país estudado (tamanho da população rural, da população pobre etc.).

Ao lado de um levantamento das vantagens e limitações de outras escalas de estratificação social para o estudo das atividades rotineiras, inclusive aquelas que não auferem rendimentos, procuramos observar as contribuições mais clássicas da Sociologia ao estudo da estratificação residencial. Apresentados os procedimentos para elaborar e validar a escala construída, buscamos, a partir dela, delinear os estratos e ressaltar como diferentes grupamentos de homens e mulheres realizam atividades domésticas na vida cotidiana.

\section{VANTAGENS E LIMITAÇÕES DAS ESCALAS DE ESTRATIFICAÇÃO SOCIAL PARA A ATRIBUIÇÃO DE VALOR ÀS OCUPAÇÕES COMO UM TODO}

Partimos da hipótese de que as principais estratégias de mensuração da posição social, que partem dos ofícios exercidos, inibem a atribuição de valor às atividades não geradoras de renda. Na pesquisa sociológica, tal dificuldade emerge no momento em que as atividades economicamente rentáveis são estratificadas por meio da aplicação de medidas que levam em consideração apenas os valores médios de renda e educação das ocupações da população estudada ponderados pela idade (Duncan, 1961:109-138; Blau, Duncan e Tyree, 1994:204). No passado, avaliava-se a posição dos ofícios no sistema de estratificação social a partir de estimativas sobre seu prestígio fornecidas pela população estudada, método também utilizado no Brasil (Hutchinson e Castaldi, 1960:19-51). Entendemos que as medidas de prestígio podem constituir um excelente recurso para estimar a posição social do trabalho das mulheres, inclusive o não remunerado, pois avaliações da importância ou do grau de deferência consignado a determinadas atividades são independentes do rendimento que auferem (Aguiar, 1980:224-238). Todavia, os estudos sobre prestígio social são limitados, porque apenas um número restrito de atividades pode ser apresentado para avaliação em um survey.

Por outro lado, as pesquisas que introduziram em seus protocolos de investigação níveis médios de educação e de rendimento das atividades ponderados por idade, além da capacidade de prever o prestígio ocupacional, oferecem a vantagem de atribuir uma cifra numérica a 
um conjunto muito mais amplo de atividades que as medidas de reputação, alcançando resultados empíricos mais abrangentes (Featherman e Hauser, 1994:219-220) - exceção feita aos ofícios excluídos do mercado de trabalho. Para o conjunto de surveys que conduzimos em Belo Horizonte (Pesquisa da Região Metropolitana de Belo Horizonte, realizada em 2002, 2005 e 2008), inclusive sobre o uso do tempo (Pesquisa dos Usos do Tempo de Belo Horizonte, realizada em 2001), empregamos uma escala não publicada, semelhante à de Duncan, calculada por Nelson do Valle Silva (1988) para o Brasil da década de 1980 e posteriormente atualizada para a Pesquisa Nacional por Amostra de Domicílios (PNAD) dos anos 1990. Os cálculos foram gentilmente cedidos por aquele autor para introdução em nossa base de dados. Alternativamente, no banco da pesquisa, empregamos um índice internacional, o International Socioeconomic Index (ISEI), elaborado por Harry Ganzeboom, Paul M. de Graaf e Donald Treiman (1992). O ISEI é calculado com fundamento na relação de atividades com remuneração desenvolvida pela Organização Internacional do Trabalho (OIT) em várias ondas sucessivas. Utilizando esses recursos, cada ocupação passou a ser graduada por uma escala que resume a posição social de seu detentor. Quanto às atividades sem receita econômica, por receberem um 0 (zero) no quesito "rendimentos do trabalho", não foi possível granjear-lhes um valor.

As medidas de estratificação social são essenciais para a análise da sociedade brasileira, detentora de altos índices de desigualdade social. As que existem até agora foram produzidas com dados da PNAD. Essa fonte de dados, todavia, traz alguns complicadores para o emprego de uma escala de estratificação ocupacional. A ampliação da listagem de ocupações realizada pelo Instituto Brasileiro de Geografia e Estatística (IBGE), dentre outras mudanças, diversificou ainda mais a relação nacional dos ofícios, elevando seu registro em um dígito. Assim, os recursos de mensuração das disparidades sociais anteriormente empregados tornaram-se defasados, criando outros desafios. Foram necessários novos cálculos sobre a posição social das ocupações para a atualização da escala. Acrescentamos-lhe mais um complicador: a proporção de mulheres que têm na ocupação de dona de casa sua atividade principal tem diminuído (Aguiar, Neves e Fernandes, 2007:167-169). Mensurar o valor dessa redução torna-se difícil se deixarmos essa ocupação de fora dos indicadores que podem aferir sua importância social. Ao incluirmos escalas avaliando as disparidades quanto ao uso do tempo, surgem algumas indagações: como atribuir

DADOS - Revista de Ciências Sociais, Rio de Janeiro, vol. 60, nº 2, 2017 
valor às atividades sem rendimentos? Como verificar as mudanças de uma condição familiar com alta proporção de donas de casa para outra em que a maioria das mulheres casadas passa a trabalhar fora? Tais questões conduzem à busca de novos indicadores, como a escala de estratificação residencial a qual discutiremos mais adiante.

A valoração do trabalho doméstico é uma questão presente na ordem do dia dos estudos de uso do tempo que buscam estimar sua contribuição ao Produto Interno Bruto (PIB) das nações. Isso é feito por meio da elaboração de contas-satélite que levam em consideração a participação do trabalho doméstico não remunerado, perspectiva construída a partir das conferências mundiais de mulheres. Duncan Ironmonger e Faye Soupourmas (2009), que fazem esse cálculo para o caso da Austrália, creditam a Andrew Harvey e Arnab Mukhopadhyay (1996) a inovação de atribuir um valor à produção doméstica com base em pesquisas de uso do tempo.

Com o olhar de sociólogos, faremos um breve percurso por essa estratégia de valoração do trabalho doméstico não remunerado à procura de um procedimento similar em outra disciplina. Buscaremos respostas a um problema comum: o que nos seria possível apreender sobre a valoração das atividades sem renda ao examinarmos as contribuições do campo da Economia Doméstica? Que perguntas lhes dirigir a partir de uma perspectiva da Estratificação Social? Existiriam pressupostos subjacentes de desigualdades sociais nas estratégias de valoração do trabalho não pago pela Economia Doméstica?

Com a busca pelo suporte de um campo de conhecimento distinto do nosso, não visamos realizar uma exegese de todas as inovações nesse domínio, mas efetuar um levantamento daquelas que incluem dimensões estratificadas subjacentes às medidas de valoração. Os pressupostos mencionados anteriormente interferem na adoção de estratégias de mensuração semelhantes para os países em desenvolvimento, como é o caso do Brasil. Observamos, assim, a construção de uma bateria de indicadores que condicionam a produção de bens por atividades não remuneradas realizadas nos domicílios.

\section{VALORAÇÃO DO TRABALHO NÃO MERCANTIL PELA PRODUÇÃO DOMÉSTICA}

Nas apreciações da Economia Doméstica, citamos dois pontos de vista principais sobre a produção doméstica: (1) o que enfoca insumos para 
essa produção (inputs) e (2) o que destaca seu produto final (outputs). A aplicabilidade desses pontos de vista aos países em desenvolvimento foi discutida por Abishek Kulshreshtha e Gulab Singh (1999:3-5), e por Julia Velazco e Jackeline Velazco (2013:21-26). Um primeiro aspecto observado por esses autores consiste em contabilizar os bens e serviços elaborados em casa, o consumo de capital que essa atividade efetua e o valor dos insumos intermediários envolvidos nessa confecção, assim como os impostos a ela consignáveis. Um segundo aspecto se concentra na pontuação dos bens e serviços produzidos no contexto domésti$\mathrm{co}$, análogos àqueles adquiríveis no mercado. Em seguida, atribui-se um valor aos objetos confeccionados em casa, equiparável aos que são produzidos para o mercado.

Os estudos do primeiro tipo geralmente se baseiam nas pesquisas de uso do tempo com o fim de calcular a quantidade de trabalho envolvida na produção não remunerada e, então, atribuir-lhe uma estimativa de custo com base em um trabalho equivalente efetuado no mercado. Há discussões na literatura acerca do padrão salarial de referência definido para essa valoração (Velazco e Velazco, 2013:23-26). Para alguns, a medida deve ser a de um salário mínimo vigente no país estudado; para outros, o salário de uma/um empregada/o doméstica/o (generalista ou especialista); outros, ainda, pleiteiam considerar atividades que nem sempre estão presentes nos países em desenvolvimento, como o da substituta da dona de casa, enquanto atividade paga pelo seguro social, alocada à administração do lar em casos de incapacitação da titular (Goldschmidt-Clermont, 1993:427-429). O exemplo alerta para a existência de diferenças internacionais entre as especialidades presentes no mercado e as atividades das donas de casa.

Outros enfoques propõem que a forma mais adequada de efetuar esse cálculo consistiria em considerar custos e oportunidades existentes para a realização das atividades domiciliares não remuneradas com base no salário que o trabalhador ou trabalhadora obtém regularmente com a venda de sua força de trabalho, levando-se em conta o tempo empregado para o exercício das tarefas domésticas (Kulshreshtha e Singh, 1999). Nesse enfoque, a título de exemplo, o valor monetário do trabalho de um dentista que também desempenhe atividades domésticas é considerado superior ao de uma balconista que desenvolva o mesmo tipo de trabalho no contexto residencial. Velazco e Velazco (2013:30-31) apontam que a perspectiva de custo e oportunidade pode alçar os valores do trabalho não remunerado a patamares muito mais

DADOS - Revista de Ciências Sociais, Rio de Janeiro, vol. 60, n’ 2, 2017 
elevados que aquela que toma o emprego doméstico como referência. Documentado com dados do Canadá e dos Estados Unidos, esse estudo demonstra que a estratégia empregada para mensurar o valor do trabalho doméstico tomando a atividade de mercado como parâmetro de equivalência ao trabalho não remunerado encontra resultados imensamente variáveis. Luisella Goldschmidt-Clermont (1993:422) avalia que esse enfoque é mais apropriado para o estudo das classes médias altas de países desenvolvidos e, reconhecendo as dificuldades da comparação internacional do critério dos custos e oportunidades, recomenda que ele não seja empregado nessa modalidade de estudos.

Sendo assim, embora a análise de custo e oportunidade se aproxime mais das preocupações dos estudos de Estratificação Social, seu uso cria dificuldades para o estabelecimento de uma estimativa padronizada para o preço médio do trabalho avaliado. Essa perspectiva parece se associar à divisão desigual do trabalho doméstico em países como o Brasil, onde as mulheres recebem remunerações menores que a dos homens para trabalhos equivalentes, havendo ainda, quando ambos trabalham fora, diferenças salariais geralmente superiores para os maridos.

Kulshreshtha e Singh (1999:9) apontam também a dificuldade de estabelecer comparações entre o valor consignado ao trabalho doméstico em países desenvolvidos e em países em desenvolvimento, já que nos últimos há uma ampla proporção de trabalho não remunerado para além do doméstico - o que se verifica, com frequência, quando há uma vasta parcela da população instalada no campo. A solução apontada por eles, ao destacarem os obstáculos à comparabilidade entre os dois âmbitos sociais, consiste em efetuar cálculos distintos para cada um dos contextos: urbano e rural. Nos países em desenvolvimento, onde o volume de trabalho não remunerado disponível é extenso, decisões sobre o valor de referência a ser empregado para o cálculo de suas contribuições ao PIB podem se tornar muito complexas, o que resulta em problemas de comparabilidade com o PIB doméstico de outras nações. $\mathrm{O}$ volume de trabalho não pago pode representar estimativas muito elevadas do PIB satélite, levando a debates sobre o valor obtido. A discussão contém suposições que atribuímos às desigualdades sociais no que diz respeito às diferenças internas presentes em países com grande população rural pobre, muito diferenciada de regiões urbanas, mais ricas e desenvolvidas. 
Ironmonger (1996) dedicou-se à conceituação e mensuração do PIB satélite de várias nações complexas, tais como a Austrália. A Economia Doméstica é por ele definida como o conjunto das atividades efetuadas por grupos domiciliares para produzir bens e serviços em uma determinada área geográfica, empregando capital doméstico e trabalho não pago, para uso próprio ou de seus familiares. Isso inclui a produção de alimentos, a lavagem de roupa e utensílios, a limpeza da casa, o cuidado com crianças, a realização de compras, de reparos, transporte, manutenção da casa, hospedagem, jardinagem, entre outras tarefas não remuneradas (ver também Goldschmidt-Clermont, 1993:421).

Vale a pena situar a proposta de Ironmonger dentro de um contexto teórico mais amplo. Sua estratégia remete aos antigos esforços da teoria marxista, como aquele efetuado por Claude Meillassoux (1979), para postular um modo de produção doméstico. Também a literatura marxista e feminista promoveu debates sobre o estatuto teórico do trabalho não pago em relação à produção capitalista nos anos setenta e oitenta, cujo ponto de partida se deve ao trabalho de Mariarosa Dalla Costa e Selma James (1972), as quais reivindicavam um salário para as atividades domésticas, chamando atenção, por exemplo, para a economia gerada pelo trabalho não pago em lugar do consumo fora de casa, como refeições preparadas em casa comparadas às adquiridas em restaurantes ou outros estabelecimentos comerciais. Essa demanda salarial gerou intensa discussão no âmbito do feminismo marxista (Seccombe, 1975; Coulson, Magas e Wainright, 1975; Molyneux, 1979; Miles, 1983), o qual concluiu que o trabalho doméstico gera valor, embora não gere mais-valia (fato não representado apenas pela reprodução da força de trabalho, mas pela produção efetuada pelas mulheres, em casa, sem remuneração).

Ironmonger, contudo, não observa a economia doméstica como uma instância separada e independente da economia capitalista, conforme pressupõem as velhas teorizações sobre o modo de produção doméstico (Meillassoux, 1979). De fato, adverte Ironmonger, a elaboração de bens em casa demanda um capital próprio. Essa produção está, portanto, interligada ao sistema capitalista. Na sua perspectiva, da mesma forma que na economia de mercado se tem em consideração (a) a quantidade de capital investido nos meios de produção e (b) o montante de trabalho alocado na transformação da matéria-prima em produto final, para fins analíticos é possível considerar a transformação doméstica de insumos convertidos em bens para o autoconsumo. Daí a

DADOS - Revista de Ciências Sociais, Rio de Janeiro, vol. 60, nº 2, 2017 
importância das pesquisas de uso do tempo, que possibilitam criar inovações nesse campo de conhecimento ao admitir a quantificação do tempo de trabalho não pago empregado na transformação dos bens realizada em domicílio. O valor da produção em casa é fruto do tempo de trabalho empregado na produção dos bens elaborados em tal contexto, adicionado ao valor do investimento utilizado nessa transformação. Ironmonger e Soupourmas (2009:14) consideram bens de capital para a produção doméstica: o domicílio (seu valor de aquisição ou de aluguel), o automóvel, os móveis e utensílios (como geladeira, forno de micro-ondas, máquina de lavar roupa), a matéria prima utilizada e o tempo de trabalho ao qual se imputa um valor para calcular a sua contribuição ao PIB.

Para aferição das atividades domésticas, no que se refere a sua inclusão nas contas-satélite do PIB, foram criadas seis categorias especiais, relacionadas a acomodações, alimentos, limpeza de roupas, cuidados com crianças, transportes, atividades voluntárias e educação. Essa conceituação implica a reconsideração das contas nacionais. Os autores avaliam que a economia doméstica da Austrália contribui com cerca de um terço a mais para o PIB do que a economia de mercado. Já os cálculos realizados para o Brasil por Hildete Melo, Claudio Considera e Alberto Di Sabbato (2007) encontraram acréscimos ao PIB da ordem de $12,76 \%$, com base em dados das PNADs. Essas informações são obtidas através de perguntas sobre o tempo dedicado aos "afazeres domésticos" e são menos específicas do que aquelas que levantam o trabalho não pago por meio de diários.

Ironmonger e Soupourmas (2009) não consideram apenas a duração dos afazeres domésticos, incluindo também em suas estimativas: o tempo gasto com a direção de um transporte (como, por exemplo, para as compras de supermercado), com o trabalho voluntário, o tempo dedicado à educação (como a supervisão dos deveres de casa das crianças), além daquilo que denominam de capital doméstico. A lista engloba, portanto, mais atividades que aquelas levantadas pelas PNADs. Importante notar, para os estudos de estratificação residencial, que os bens enumerados como componentes do capital doméstico poderiam integrar uma escala de estratificação residencial.

Encontramos, portanto, duas pontes entre os estudos que buscam valorar o trabalho não remunerado no campo da Economia Doméstica e no da Estratificação Social em Sociologia. A primeira diz respeito às 
Estratificação Residencial, Valoração do Trabalho Doméstico e Uso do Tempo

atividades de mercado que são associadas, pelos estudiosos, ao trabalho doméstico (tema que, como visto acima, tem gerado muitas controvérsias). A segunda ponte diz respeito aos bens de capital que entram no cálculo do PIB doméstico, parte que interessa mais ao presente trabalho, podendo ser utilizados para estratificar os domicílios. Como queríamos observar se haveria diferenças no uso do tempo entre homens e mulheres que ocupam diferentes posições sociais, não incluímos dados sobre o tempo de trabalho não remunerado em nossa Escala de Estratificação Residencial, como fizeram Ironmonger e Sopournas (2009) quando buscaram estimar um valor em seu cálculo do PIB doméstico. No nosso caso, o tempo devotado às atividades domésticas compreende a nossa variável dependente (ver Tabela 1, mais adiante) e observamos sua correlação com um conjunto de bens presentes nos domicílios, conformando uma escala.

\section{O APORTE EMPÍRICO DO CAMPO DA ESTRATIFICAÇÃO SOCIAL: BENS DOMICILIARES COMO INDICADORES DA DESIGUALDADE SOCIAL}

Os primeiros estudos de Estratificação Social utilizaram componentes das residências como elementos de diferenciação dos grupos domésticos que nelas residiam. William Sewell (1940) realizou um levantamento dessas estratégias para a construção de uma escala padronizada, posteriormente empregada por ele próprio e por seus seguidores em vários estudos empíricos, como em Oklahoma, nos Estados Unidos, e também num município do interior de Minas Gerais, no Brasil (Saraiva,1969; Haller e Saraiva, 1972; Haller e Haller, 2009).

Em seu trabalho, Sewell (1940) delibera que para indicar o status socioeconômico de indivíduos e famílias no contexto rural é importante observar a posse de bens materiais (habitação, equipamentos e componentes da residência), de bens culturais e a participação em atividades comunitárias. A casa, a propriedade e seus equipamentos são enumerados perfazendo um total de 66 itens $^{1}$. A lista compreende ainda a presença de empregados domésticos e características da educação, cultura e de participação voluntária em clubes, igrejas, cooperativas e em atividades recreativas da esposa e do marido, atingindo um total de 123 categorias. A relação abrange, além disso, informações acerca do número de respondentes à questão sobre a posse do bem, a percentagem de respondentes, o número de pessoas de posse do item e o seu percentual. Em seguida, o autor observa o grau de correlação entre as respostas obtidas e o grau de consistência interna entre elas. Dividindo

DADOS - Revista de Ciências Sociais, Rio de Janeiro, vol. 60, nº 2, 2017 
a população em quartis, analisa, então, a percentagem de ocorrência dos itens pelo conjunto da população, além de avaliar a capacidade de cada um deles para estabelecer diferenças entre os quartis. O procedimento resulta na eliminação de 82 elementos, restando 36 componentes, ponderados e padronizados para compor uma escala (Sewell, 1940:42). A medida é então validada com base em sua capacidade de diferenciar entre proprietários, arrendatários, hóspedes e trabalhadores, e seu grau de confiabilidade é verificado através de três métodos diversos, de modo a satisfazer os padrões usuais das escalas de estratificação social. Consideramos ser o método de Sewell um precursor do adotado no presente artigo.

Uma versão desse conjunto, com 24 itens, foi adaptada por Hélcio Saraiva (Saraiva, 1969; Haller e Saraiva, 1972) para uma região rural do Brasil, sendo validada como medida de estratificação por Haller e Haller (2009). Suas metodologias, ao incorporar os trabalhos prestados à residência em conjunto com o capital doméstico nela presente, seguem a estratégia de Sewell. Os serviços considerados incluem a presença de cozinheira, lavadeira e passadeira, implicitamente atribuindo status mais baixo àquelas famílias que não as podem contratar. As medidas incorporam, ainda, três itens de uso próprio e apresentação social (possuir terno, relógio e o tipo de calçado usado na entrevista). Os demais componentes abarcam características da construção da residência, processados segundo o método de Sewell, observando sua capacidade de diferenciar a população ${ }^{2}$. Haller e Haller (2009) concluem que tais medidas estão altamente associadas com quatro componentes do sistema de mensuração das desigualdades, em suas dimensões econômica, social, política e educacional, tendo sido constatada sua relevância para a teoria da estratificação social que ambos os autores privilegiam.

Escalas para mensurar o status social foram primeiramente disseminadas com base em estudos do prestígio das ocupações. Tais mensurações foram subsequentemente generalizadas a partir do nível educacional e da renda gerada por essas atividades, possibilitando abarcar um número bem mais amplo do que as medidas de reputação, que recobrem uma quantidade limitada de casos. Essa estratégia, contudo, limitou-se à estimativa de status dos trabalhos com rendimentos, deixando sem posição social própria as donas de casa e os estudantes, dentre outras funções sem proventos. A proposta de uma escala de estratificação residencial supre a possibilidade de atribuir um status a 
todo e qualquer corresidente de um grupo doméstico ao qual se atribui uma posição na EER. O domicílio e seus equipamentos recebem contribuições diversas de seus moradores para adquiri-los, alugá-los e mantê-los, podendo resumir a posição social de seus residentes.

\section{Fonte de Dados}

São utilizados os dados da pesquisa "Múltiplas Temporalidades de Referência: Pesquisa de Uso do Tempo por Amostragem Domiciliar", realizada em 2001 no município de Belo Horizonte, a partir de uma amostra probabilística de 371 domicílios. Cada membro acima de 8 anos de idade preencheu um diário de uso do tempo para um dia da semana (num total de 1.124 diários) e outro para um dia de final de semana (num total de 1.133 diários), sendo que 7,5\% deles foram especialmente desenhados para a população de baixa escolaridade.

Além das informações sobre o cotidiano de seus membros, obteve-se, para cada residência, dados acerca de suas características socioeconômicas e demográficas, assim como sobre a estrutura domiciliar e a posse de bens duráveis, a partir dos quais foi construída uma escala que diferencia a população estudada.

\section{A ESCALA DE ESTRATIFICAÇÃO RESIDENCIAL}

A partir de informações fornecidas pela população amostrada, pode-se avaliar uma lista extensa de possíveis componentes da escala de status. Para identificar os itens que melhor contribuiriam para a sua construção, empregou-se a Teoria da Resposta ao Item (TRI) para a pesquisa de Belo Horizonte e para a Pesquisa de Orçamento Familiar (POF) de 2009, conduzida pelo IBGE, utilizada para validar a EER. A TRI permite identificar a dimensão latente da estratificação social presente na amostra estudada (Baker, 2001), possibilitando diferenciá-la em estratos, mesmo quando os indicadores mais frequentemente usados na literatura sobre o tema, como renda individual ou rendimentos da ocupação, encontram-se ausentes (Soares, 2005). A TRI possibilita incorporar dados sobre um item determinado, mesmo que haja incompletudes e lacunas informativas. Essa teoria vem sendo extensamente utilizada há várias décadas em pesquisas no campo educacional, e seu emprego em outras áreas de investigação social, como argumentam Alves e Soares (2009), pode ajudar a superar obstáculos que envolvam a construção de indicadores socioeconômicos.

DADOS - Revista de Ciências Sociais, Rio de Janeiro, vol. 60, n’ 2, 2017 
Utilizamos um modelo misto, apropriado para a construção de escalas que envolvam, ao mesmo tempo, variáveis binárias e categóricas/ordinais - para estas últimas foi empregado o método proposto por Samejima (1968) - com o emprego do software XCalibre. A partir desse modelo, a capacidade de distinção de cada item na amostra pôde ser avaliada, o que permitiu incluir, na escala final, apenas aqueles que mais diferenciaram a população estudada.

Com os recursos da TRI verificou-se que, entre todos os itens avaliados, aqueles com melhor poder de discriminação, e que foram mantidos para a construção da escala, eram: máquina de lavar roupas, máquina de lavar louças, aparelho de micro-ondas, aspirador de pó, refrigerador, automóvel, telefone fixo, televisão em cores, computador, número de banheiros, aparelho de videocassete/DVD, qualidade do piso e número de cômodos per capita. A matriz de correlação policórica para esses itens pode ser verificada no Apêndice A e, em linhas gerais, a informação mais importante a se extrair dela é a correlação positiva entre todos eles.

Itens como a propriedade do domicílio, o tipo de coleta de lixo utilizada, a existência de telefone celular, o tipo de casa, seu estado de manutenção e a sua localização, que haviam sido previamente considerados, foram descartados por diferenciarem pouco a população estudada. Um exemplo sobre o pequeno poder de diferenciação da propriedade domiciliar pode ser relacionado ao entendimento da pergunta efetuada para avaliar essa condição: residentes em zonas de invasão podem responder que são proprietários de seus domicílios, o mesmo ocorrendo com adquirentes de imóveis e com donos de residências abastadas, quando todos se descrevem dessa mesma maneira.

O poder de diferenciação dos itens é facilmente visualizado nos gráficos a seguir, que apresentam a curva característica de dois itens que selecionamos entre aqueles que foram inicialmente considerados. O Gráfico 1 mostra esse poder para a posse de máquina de lavar roupas, por exemplo. Pelo gráfico, a ausência de máquina de lavar roupas é indicada pela curva 1, enquanto a posse de tanquinho é observada pela curva 2 e a posse de uma máquina completa é descrita pela curva 3 . Note-se que à medida que aumenta a escala de status (eixo horizontal), aumenta também a probabilidade de posse de uma máquina de lavar, comprovando que esse é um bom elemento para a composição da nossa escala. Da mesma forma, o indicador de detenção de uma geladeira 


\section{Gráfico 1}

Distribuição do Poder de Diferenciação do Item “Máquina de Lavar" na Escala de Status Residencial

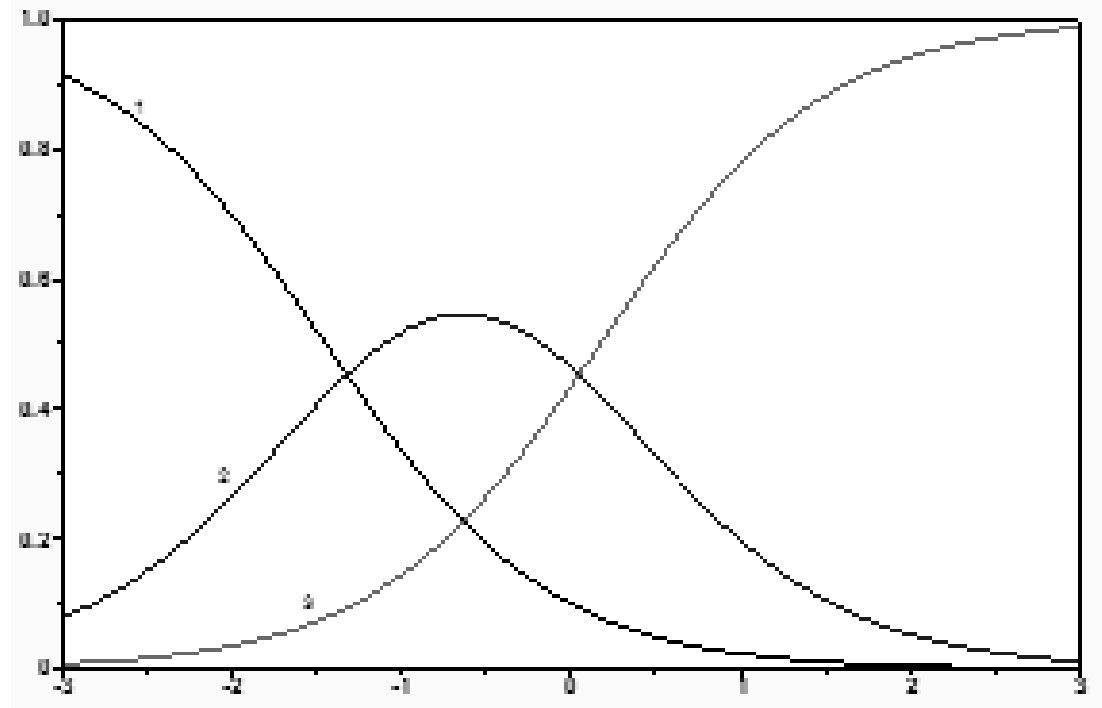

Fonte: Elaboração própria a partir de dados da Pesquisa de Usos do Tempo. Obs.: 1) sem máquina de lavar; 2) tanquinho; 3) máquina de lavar.

permite a identificação daqueles que possuem um nível de status muito baixo e o que indica a posse de um ou mais carros por aqueles com status mais elevado.

Os itens dotados de pouca variabilidade junto à população se prestam pouco à construção da escala, sendo então excluídos. Seu fraco poder de diferenciação também é facilmente visualizado no mesmo tipo de gráfico, como ilustra o Gráfico 2 para "propriedade do domicílio". Como pode ser observado, três (domicílios cedidos, alugados e outras condições) das quatro categorias (a quarta indica domicílio próprio) não apresentam diferenças entre si à medida que a escala aumenta, indicando que diferenças internas nesta variável não contribuem muito para a diferenciação dos domicílios.

A maioria dos itens mantidos para a escala final se encaixa principalmente naquilo que Ironmonger e Soupourmas (2009:14) denominaram de capital do domicílio. Embora um computador possa ser utilizado para realizar trabalho remunerado em casa, ele pode também ser usado para administração da residência. $\mathrm{O}$ mesmo pode ser dito sobre a posse de um telefone fixo e de outros equipamentos.

DADOS - Revista de Ciências Sociais, Rio de Janeiro, vol. 60, n² 2, 2017 
Gráfico 2

Distribuição do Poder de Diferenciação do Item "Propriedade do Domicílio" na Escala de Status Residencial

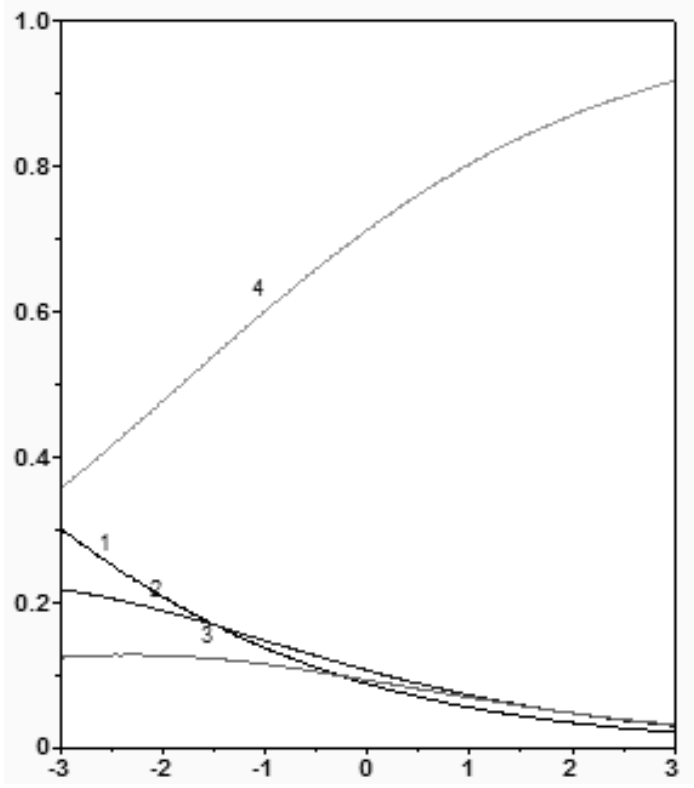

Fonte: Elaboração própria a partir de dados da Pesquisa de Usos do Tempo.

Obs.: 1) Domicílio cedido; 2) domicílio alugado; 3) outros tipos de posse; 4) domicílio próprio.

A escala, em seu formato original, é padronizada, com média 0 e desvio padrão 1, valor mínimo igual a -3 e máximo igual a 3. A escala é posteriormente transformada para ter apenas valores positivos, variando entre 0 e 100. Sua distribuição pode ser visualizada no histograma abaixo (Gráfico 3), indicando que perfaz uma curva aproximadamente normal.

Para confirmar a robustez do instrumento, uma escala com os mesmos itens foi construída com dados de aproximadamente 43 mil domicílios urbanos entrevistados pela Pesquisa de Orçamento Familiar (POF) de 2008-2009, coletada pelo IBGE . A correlação entre as duas escalas foi altamente positiva: 0,983 $(p<0,001)$. Além disso, estimamos também que a correlação entre a EER e a escala de status socioeconômico produzida por Silva (1988) para o mercado de trabalho brasileiro é de 0,496 ( $p$ $<0,001$ ) e a obtida em conformidade com o ISEI (Ganzeboom, De Graaf e Treiman, 1992) é de 0,534 ( $p<0,001)$. Esses coeficientes, de magnitude moderada a alta, indicam que a escala varia significativamente na mesma direção de escalas consolidadas de status socioeconômico, e é um sólido indicador de status socioeconômico do domicílio. 
Gráfico 3

Distribuição da Escala de Status Residencial

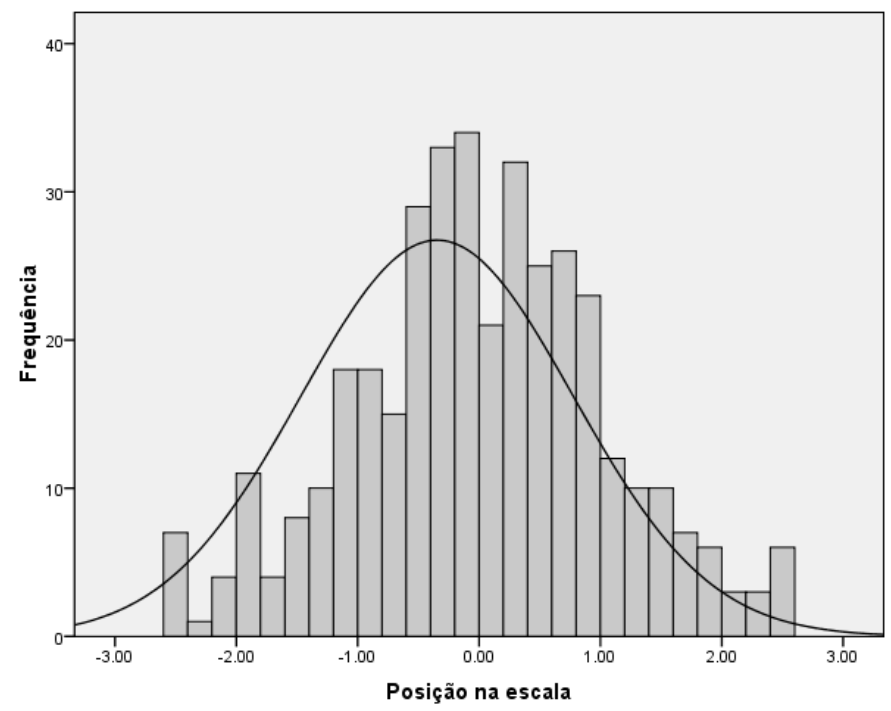

Fonte: Elaboração própria a partir de dados da Pesquisa de Usos do Tempo.

\section{Estratificação Residencial e Trabalho Doméstico Não Remunerado}

A Tabela 1 traz informações sobre tempo médio de dedicação aos cuidados com a casa e a família por sexo e status residencial (quartis), durante os dias de semana e fim de semana. Traz também os coeficientes de correlação entre tempo de dedicação aos cuidados domésticos e a escala de status residencial.

Na Tabela 1 se observa que as mulheres despendem mais que o dobro do tempo gasto por homens com os cuidados com a casa e a família durante os dias de semana e quase o dobro num dia de final de semana. Elas reduzem o tempo de atividades domésticas no final de semana, enquanto os homens, no mesmo período, elevam relativamente esse tempo.

Há uma baixa correlação negativa entre o status residencial e o desempenho das atividades domésticas: $-0,07$ durante a semana e $-0,01$ no fim de semana $(p<0,01)$. Isso quer dizer que a divisão sexual do trabalho é acentuada em todas as camadas sociais. Outra observação permite constatar que as distinções mensuradas implicam em diferenças no tempo dedicado ao trabalho doméstico. Em média, quanto mais alto o

DADOS - Revista de Ciências Sociais, Rio de Janeiro, vol. 60, n² 2, 2017 
Tabela 1

Tempo Médio (em minutos) Dedicado ao Trabalho Doméstico, por Sexo e Status Residencial

\begin{tabular}{|c|c|c|}
\hline Grupos de Análise & Dia de Semana & Fim de Semana \\
\hline \multicolumn{3}{|l|}{ Sexo } \\
\hline Homens & 115 & 151 \\
\hline Mulheres & 274 & 267 \\
\hline \multicolumn{3}{|l|}{ Status residencial } \\
\hline Quartil 1 (base da escala) & 298 & 272 \\
\hline Quartil 2 (meio - inferior) & 254 & 247 \\
\hline Quartil 3 (meio - superior) & 273 & 248 \\
\hline Quartil 4 (topo da escala) & 148 & 224 \\
\hline Correlação & $-0,07^{*}$ & $-0,01^{*}$ \\
\hline \multicolumn{3}{|l|}{ Sexo $\times$ Status residencial } \\
\hline \multicolumn{3}{|l|}{ Homens } \\
\hline Quartil 1 & 79 & 54 \\
\hline Quartil 2 & 209 & 156 \\
\hline Quartil 3 & 125 & 180 \\
\hline Quartil 4 & 38 & 181 \\
\hline Correlação & $-0,16^{*}$ & $0,05^{*}$ \\
\hline \multicolumn{3}{|l|}{ Mulheres } \\
\hline Quartil 1 & 313 & 286 \\
\hline Quartil 2 & 262 & 262 \\
\hline Quartil 3 & 301 & 260 \\
\hline Quartil 4 & 182 & 238 \\
\hline Correlação & $-0,05^{*}$ & $-0,05^{*}$ \\
\hline
\end{tabular}

Fonte: Elaboração própria a partir de dados da Pesquisa de Usos do Tempo. ${ }^{*} p<0,01$.

status, menor o tempo dedicado a esses cuidados, tanto num dia de semana quanto no final de semana.

Para entender melhor essas diferenças, associamos status residencial e gênero, verificando que enquanto a correlação negativa é válida para mulheres, tanto na semana quanto no fim de semana, isto é, quanto menor o status residencial maior a quantidade de tempo devotada ao trabalho doméstico, para os homens há dois padrões diferentes. Em dias de semana, a correlação também é negativa. No entanto, ela é quadráti$\mathrm{ca}$, aumentando à medida que atinge o ponto médio da distribuição, $\mathrm{e}$ diminuindo a partir daí. Nos fins de semana, por outro lado, a correla- 
ção é positiva, de forma que quanto maior o status residencial, maior o tempo gasto em trabalhos domésticos.

Podemos associar esse padrão diferencial de desempenho das atividades domésticas a uma análise recentemente efetuada por Monique Meron (2014) para o caso da França. Ela identifica que as mudanças no exercício do trabalho não remunerado apresentam pelo menos dois ciclos na divisão sexual do trabalho: (1) mudança estrutural e (2) mudança comportamental (ver também Champagne, Pailhé e Solaz, 2014). Associa-se o que ela denomina de mudança estrutural ao mesmo tipo de processo identificado para um período de 28 anos (entre 1973 e 2001) em relação ao caso do Brasil (Aguiar, 2011:82-85), quando o trabalho doméstico de homens e mulheres se reduziu, o que se atribui à redução do tamanho das unidades domésticas. Com o controle da fecundidade, as mulheres passaram a ter menos filhos e assim o tamanho das famílias diminuiu e, por consequência, a duração das atividades domésticas e dos cuidados com as crianças também ficou menor.

Já o segundo fenômeno, apontado por Clara Champagne, Ariane Pailhé e Anne Solaz (2014) e por Meron (2014), é pouco perceptível na sociedade brasileira, pois as autoras se referem à introdução de uma série de equipamentos domésticos no conjunto da sociedade francesa que diminuíram o montante das tarefas domésticas e possibilitaram seu emprego pelos homens. Ou seja, o estilo de vida se transformou. Podemos, todavia, usar algumas pistas sobre o tema, levantadas pela Tabela 1: possivelmente os homens dos estratos mais elevados, quando permanecem mais tempo em casa, particularmente nos finais de semana, aumentem a sua participação nas atividades domésticas, fazendo uso de equipamentos mais sofisticados nesse processo. As mulheres fazem o movimento inverso, reduzindo o tempo de trabalho doméstico nos finais de semana, embora o volume de tempo que alocam a essas atividades ainda seja superior ao dos homens. Entre os fatores que modificam ligeiramente o tempo dedicado aos cuidados domésticos entre as mulheres, temos não apenas o acesso ao trabalho remunerado fora de casa, mas também a contratação de pessoas para execução de atividades domésticas (Aguiar e Suyama, 2011). Pesquisas futuras nos ajudarão a aprofundar essa questão, mas estudos recentes (Abramo e Valenzuela, 2014:290) já apontam a relação inversa entre pobreza e uso de equipamentos sofisticados na cozinha para o caso do Brasil, usando dados da Pesquisa de Orçamento Familiar. Todas essas indicações nos incentivam a prosseguir em posteriores trabalhos com dados brasilei-

DADOS - Revista de Ciências Sociais, Rio de Janeiro, vol. 60, n’ 2, 2017 
ros de uso do tempo nesse promissor caminho analítico relacionando estratificação social, gênero e divisão sexual do trabalho em dias de semana e em finais de semana.

Organismos internacionais, como a Comissão Econômica para a América Latina e o Caribe (Cepal), têm oferecido sugestões para o encaminhamento de Políticas Nacionais de Cuidados que aumentem o uso de equipamentos coletivos - como creches, escolas em tempo integral, lavanderias e restaurantes populares - possibilitando reduzir os encargos domésticos. Uma decorrência dessas políticas é a identificação de situações críticas em países da região, que possibilitem o acesso direto das donas de casa às políticas previdenciárias (Montaño e Magaña, 2010). As políticas de cuidados reduzem o tempo de trabalho não pago, embora as relações de poder na esfera doméstica, que impactam a divisão sexual do trabalho, tenham permanecido (Miles, 1983:197-209).

\section{CONCLUSÃO}

A escala de estratificação residencial que desenvolvemos no presente artigo faz parte de uma tendência mais geral, situada em várias áreas das Ciências Sociais, que consiste em possibilitar, de um lado, a valoração das atividades não remuneradas, e de outro, o estudo das desigualdades sociais e a observação da divisão sexual do trabalho por estrato social. Ao analisarmos os pressupostos da Economia Doméstica, incorporados a iniciativas semelhantes à nossa, descobrimos que a valoração do trabalho não mercantil passa pela enumeração de bens empregados na produção doméstica, tais como a própria moradia onde os produtos são transformados em alimentos e os aparelhos domésticos que lhe dão suporte (geladeira, máquina de lavar, entre outros equipamentos como o automóvel, usado para compras e transporte de familiares, e o computador, como parte do gerenciamento doméstico).

Alguns desses bens encontram-se disseminados entre todas as camadas sociais, mas há outros cuja sofisticação torna o acesso mais restrito, alcançável apenas por uma pequena parcela da sociedade. Tais disparidades precisam ser consideradas na valoração do trabalho doméstico e ocasionam dificuldades na comparação entre países. Nesse ponto, demonstramos que o acesso a esses bens diferencia a população em estratos. Em seguimento a essa linha de raciocínio, identificamos uma lista de itens, com base em uma antiga tradição sociológica, e configu- 
ramos a Escala de Estratificação Residencial (EER), validando-a ao correlacioná-la com outra escala semelhante construída com os dados da POF e com outras medidas de estratificação social.

A EER permite a valoração da ocupação de "dona de casa", apesar de tal atividade não granjear rendimentos. Em lugar de incorporarmos o tempo de trabalho não remunerado ao valor dos insumos para a produção doméstica, tomamos o tempo de trabalho não pago como nossa variável dependente. Além de constatarmos a presença da divisão sexual do trabalho em todos os estratos sociais, registramos ainda uma correlação negativa muito baixa entre status residencial e tempo de cuidados domésticos, o que indica que quanto mais baixo o estrato residencial maior o tempo devotado a essas atividades. O acesso a implementos, portanto, encurta as atividades não remuneradas enquanto diferencia a população.

Os resultados também possibilitam observar que, na medida em que estão mais disponíveis em casa, nos finais de semana, os homens dos estratos mais altos elevam o tempo de dedicação aos afazeres domésticos. Ao lado da identificação realizada em outro texto (Aguiar, 2011:82-85), segundo a qual fatores demográficos promovem a redução das atividades domésticas, podemos associar esse acréscimo do tempo de participação em tarefas domiciliares por parte dos homens dos estratos mais altos à mesma tendência retratada por Meron (2014) e por Champagne, Pailhé e Solaz (2014) para o conjunto da sociedade francesa, como uma mudança de natureza comportamental representada pela disseminação do uso de utensílios que facilitam sua utilização pelos homens e encurtam o tempo dedicado a esse tipo de afazer, embora sem acabar com a divisão sexual do trabalho. Estudos futuros apontarão, com maior clareza, tendências sobre essa relação.

Apresentamos, no presente texto, a EER, que conjugada ao tempo devotado às atividades não remuneradas permite aferir a divisão sexual do trabalho pela posição social da população estudada. Algumas contribuições que vêm associando o processo de valoração do trabalho doméstico não remunerado ao Produto Interno Bruto também fazem uso de um conjunto de variáveis referentes ao domicílio, suas acomodações e equipamentos empregados na produção doméstica, assim como indicadores sobre o tempo de trabalho não remunerado, para calcular seus aportes às contas nacionais. Trabalhar com essa escala permite tornar o trabalho doméstico visível no campo da Sociologia e, além dis-

DADOS - Revista de Ciências Sociais, Rio de Janeiro, vol. 60, nº 2, 2017 
so, observar que a valoração do trabalho não remunerado é uma tarefa pertinente a vários ramos das Ciências Sociais e não apenas ao campo da Economia Doméstica. Nossa passagem por essas áreas do conhecimento sobre o cotidiano, tão próximas e tão difíceis de serem cotejadas, nos permite, ademais, identificar que este é um campo ainda em construção, o que resulta em dificuldades de comparação com dados internacionais, embora se possa observar que a perspectiva comparada permite indicar mudanças demográficas e de estilo de vida impactantes na divisão sexual do trabalho no que diz respeito ao exercício das atividades não remuneradas no âmbito doméstico.

(Recebido para publicação em março de 2016) (Aprovado para publicação em março de 2017) 
Estratificação Residencial, Valoração do Trabalho Doméstico e Uso do Tempo

\section{NOTAS}

1. Os itens incluem: gramado, jardim com flores, tipo de construção da casa, aquecimento central, água encanada, iluminação, banheiro dentro de casa, esgoto, sala de jantar separada dos outros cômodos, sala de visitas separada dos outros cômodos, cozinha separada dos outros cômodos, número de janelas, número de cômodos pelo total de membros da família, qualidade das paredes, presença de cortinas, armários, tapetes, cadeiras de balanço, relógios, quadros, calendário, sofá, estante, rádio, máquina de lavar, refrigerador, tipo de chão da cozinha, tamanho da cozinha, ferro de passar, aspirador, máquina de costura, mesa e cadeiras da sala de jantar, livros, assinatura de jornal, revistas e automóvel.

2. Entre os itens estão: chão, paredes, teto, telhado, origem da água, origem da água de beber, tipo de esgoto, número de cômodos, existência de varanda, presença de banheiro, tipo de iluminação, número de cadeiras, rádio, máquina de costura e meio de transporte utilizado.

3. Informações adicionais sobre a escala construída com os dados da POF não foram incluídas neste artigo, mas podem ser disponibilizadas pelos autores para os leitores interessados. 


\section{REFERÊNCIAS BIBLIOGRÁFICAS}

ABRAMO, Laís; VALENZUELA, Maria Elena. (2014), Repartição Desigual do Tempo de Trabalho Remunerado e Não Remunerado na América Latina. Trabalho apresentado no Seminário Internacional Trabalho, Cuidados e Políticas Sociais: Brasil-França em Debate. São Paulo/Rio de Janeiro, USP/UFRJ/MAGE, 26-27 de agosto.

AGUIAR, Neuma. (1980), Tempo de Transformação no Nordeste. Petrópolis, Editora Vozes.

(2011), “Mudanças no Uso do Tempo na Sociedade Brasileira”. Política \& Trabalho, no 34, pp. 73-106.

AGUIAR, Neuma; NEVES, Jorge A.; FERNANDES, Danielle. (2007), “Mobilidade Social Feminina", in N. Aguiar (ed.), Desigualdades Sociais, Redes de Sociabilidade e Participação Política. Belo Horizonte, Editora UFMG, pp. 165-180.

AGUIAR, Neuma; SUYAMA, Emílio. (2011), Questões Metodológicas para o Estudo das Determinações do Uso do Tempo por Homens e Mulheres de Distintos Estratos Sociais. Trabalho apresentado no seminário Políticas de Tiempo y el Tiempo de las Políticas?. Santiago, Chile, Cepal, 29-30 de novembro.

ALVES, Maria Teresa Gonzaga; SOARES, José Francisco. (2009), “Medidas de Nível Socioeconômico em Pesquisas Sociais: Uma Aplicação aos Dados de uma Pesquisa Educacional". Opinião Pública, vol. 15, no 1, pp. 1-30.

BAKER, Frank B. (2001), The Basics of Item Response Theory. Washington, D.C., Eric Clearing House on Assessment and Evaluation.

BLAU, Peter; DUNCAN, Otis Dudley; TYREE, Andrea. (1994), "Measuring the Status of Occupations", in D. Grusky (ed.), Social Stratification in Sociological Perspective. Boulder, Westview Press, pp. 204-208.

CHAMPAGNE, Clara; PAILHÉ, Ariane; SOLAZ, Anne. (2014), “25 Ans de Participation des Hommes et des Femmes au Travail Domestique: Quells Facteurs D'Évolutions?". Documents de Travail, nำ203, Paris, Institut National D’Études Démographiques, pp. 1-36.

COULSON, Margaret; MAGAS, Branka; WAINWRIGHT, Hilary. (1975), “The Housewife and her Labour under Capitalism: A Critique". New Left Review, vol. 89, pp. 59-71.

DALLA COSTA, Mariarosa; JAMES, Selma. (1972), The Power of Women and the Subversion of the Community. Bristol, Falling Wall Press.

DUNCAN, Otis Dudley. (1961), "A Socioeconomic Index for All Occupations”, in A. J. Reiss Jr. (ed.), Occupations and Social Status. New York, The Free Press of Glencoe, pp. 109-138.

FEATHERMAN, David; HAUSER, Robert. (1994), “Prestige or Socioeconomic Scales in the Study of Occupational Achievement?", in D. Grusky (ed.), Social Stratification in Sociological Perspective. Boulder, Westview Press, pp. 219-220.

GANZEBOOM, Harry B. G.; DE GRAAF, Paul M.; TREIMAN, Donald J. (1992), “A Standard International Socioeconomic Index of Occupational Status". Social Science Research, vol. 21, pp. 1-56. 
Estratificação Residencial, Valoração do Trabalho Doméstico e Uso do Tempo

GOLDSCHMIDT-CLERMONT, Luisella. (1993), "Monetary Valuation of Non-Market Productive Time: Methodological Considerations". Review of Income and Wealth, vol. 39, no 4, pp. 419-433.

HALLER, William J.; HALLER, Archibald O. (2009), "Household Socioeconomic Status Scales: Theoretic Anomalies?". Population Review, vol. 48, no 2, pp. 66-78.

HALLER, Archibald O.; SARAIVA, Hélcio Ulhoa. (1972), "Status Measurement and the Variable Discrimination Hypothesis". Rural Sociology, vol. 37, pp. 325-351.

HARVEY, Andrew; MUKHOPADHYAY, Arnab. (1996), The Role of Time Use Studies in Measuring Household Outputs. Trabalho apresentado na Conference of the International Association for Research on Income and Wealth: Accounting for Time. Lillehammer, Noruega, International Association for Research on Income and Wealth, 18-24 de agosto.

HUTCHINSON, Bertram; CASTALDI, Carlo. (1960), "A Hierarquia de Prestígio das Ocupações", in B. Hutchinson (org.), Mobilidade e Trabalho: Um Estudo na Cidade de São Paulo. Rio de Janeiro, Centro Brasileiro de Pesquisas Educacionais e Instituto Nacional de Estudos Pedagógicos, pp. 19-51.

IRONMONGER, Duncan. (1996), “Counting Outputs, Capital Inputs and Caring Labor: Estimating Gross Household Product”. Feminist Economics, vol. 2, no 3, pp. 37-64.

; SOUPOURMAS, Faye. (2009), “Estimating Household Production Outputs with Time Use Episode Data". Electronic International Journal of Time Use Research, vol. 6, no 2, pp. 240-268.

KULSHRESHTHA, Abishek C.; SINGH, Gulab. (1999), Valuation of Non-Market Household Production. Trabalho apresentado no Seminário Internacional Time Use Studies. Ahmedabad, India, Centre for Development Alternatives, 7-10 de dezembro.

MEILLASSOUX, Claude. (1979), Femmes, Greniers et Capitaux. Paris, Maspéro.

MELO, Hildete P.; CONSIDERA, Claudio; DI SABBATO, Alberto. (2007), “Os Afazeres Domésticos Contam". Economia e Sociedade, vol. 16, no 3, pp. 435-454.

MERON, Monique. (2014), Travail Rémunéré, Travail Domestique: Evolution de la Repartition Sexuelle en France et Reflexions sur les Changements de Concepts d'Activite aux Fils du Temps. Trabalho apresentado no Seminário Internacional Trabalho, Cuidados e Políticas Sociais: Brasil-França em Debate. São Paulo/Rio de Janeiro, USP/UFRJ/MAGE, 26-27 de agosto.

MILES, Angela. (1983), "Economism and Feminism: Hidden in the Household: A Comment on the Domestic Labour Debate". Studies in Political Economy, vol. 11, no 1, pp. 197-209.

MOLYNEUX, Maxine. (1979), "Beyond the Domestic Labour Debate". New Left Review, vol. 116, pp. 3-27.

MONTAÑO, Sonia; MAGAÑA, Coral Calderón. (2010), El Cuidado en Acción: Entre el Derecho y el Trabajo. Chile, Cepal.

SAMEJIMA, Fumi. (1968), "Estimation of Latent Ability Using a Response Pattern of Graded Scores". ETS Research Bulletin Series, vol. 1, pp. i-169.

DADOS - Revista de Ciências Sociais, Rio de Janeiro, vol. 60, n² 2, 2017 


\section{Neuma Aguiar e Arnaldo Mont'Alvão}

SARAIVA, Hélcio. (1969), The Variable Discrimination Hypothesis and the Measurement of Socioeconomic Status in an Isolated Brazilian Region. Tese (Doutorado em Sociologia), Programa de Pós-Graduação em Sociologia, University of Wisconsin, Madison.

SECCOMBE, Wally. (1975), “The Housewife and Her Labor under Capitalism”. New Left Review, vol. 83, pp. 3-24.

SEWELL, William H. (1940), "The Construction and Standardization of a Scale to Measure the Socio-Economic Status of Oklahoma Farm Families". Agricultural College Technical Bulletin, no 9, pp. 1-88.

SILVA, Nelson do Valle. (1988), Uma Classificação Ocupacional para o Estudo da Mobilidade e da Situação de Trabalho no Brasil. Rio de Janeiro, LNCC, mimeo.

SOARES, Tufi Machado. (2005), “Utilização da Teoria da Resposta ao Item na Produção de Indicadores Socioeconômicos". Pesquisa Operacional, vol. 25, no 1, pp. 83-112.

VELAZCO, Julia; VELAZCO, Jackeline. (2013), “Estimación del Valor Económico del Trabajo No Remunerado: Una Aplicación para Perú", in M. Ramos (org.), El Uso del Tiempo de las Mujeres: Trabajo Gratuito de las Personas y Datos de la Encuesta Nacional de Uso del Tiempo: Dos Studios y Dos Reflexiones. Lima, Economistas Sin Fronteras, pp. 11-74. 
Estratificação Residencial, Valoração do Trabalho Doméstico e Uso do Tempo

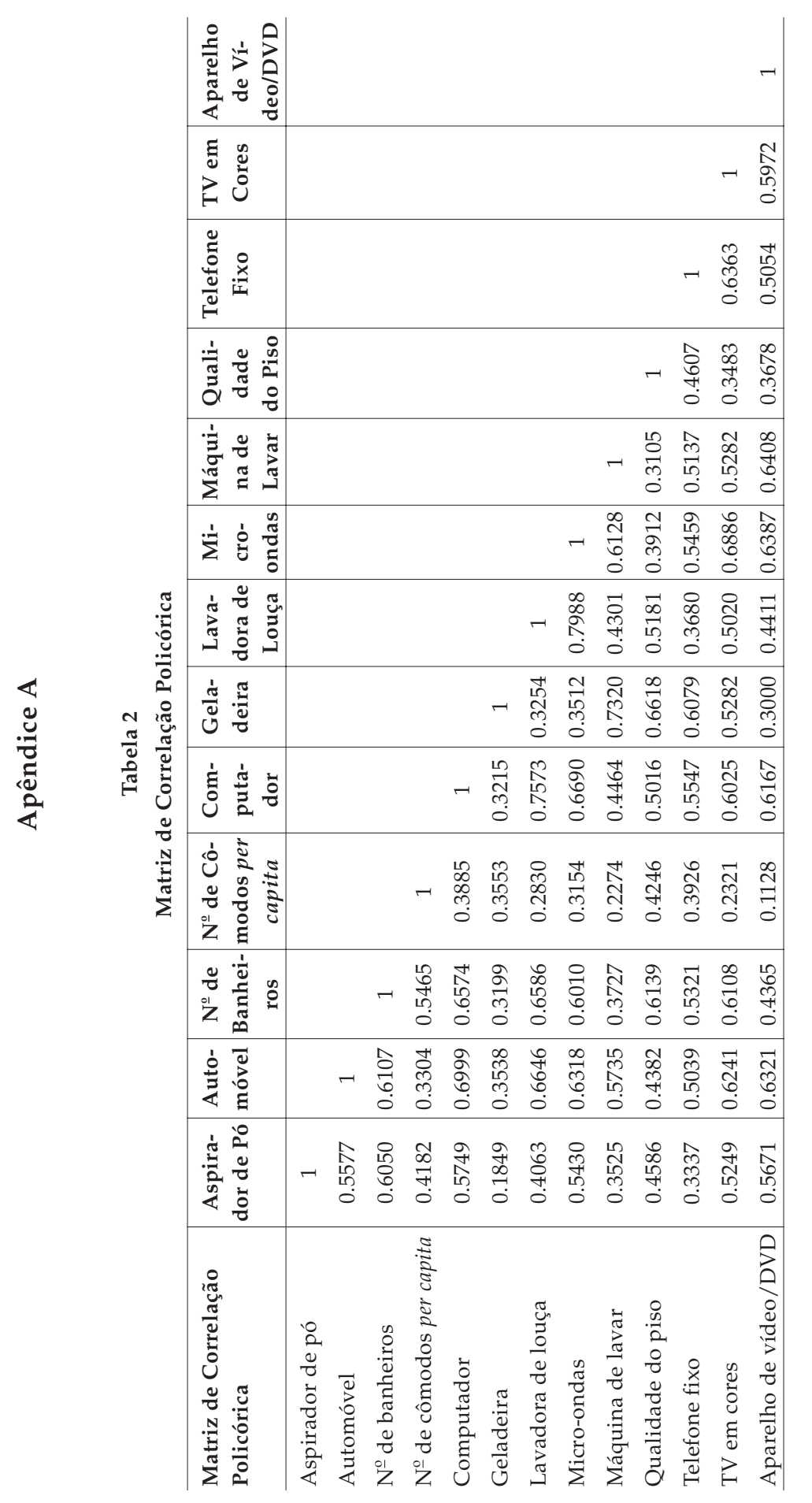

DADOS - Revista de Ciências Sociais, Rio de Janeiro, vol. 60, nํㅡ 2, 2017 
RESUMO

Estratificação Residencial, Valoração do Trabalho Doméstico e Uso do Tempo: Contribuições para a Análise do Caso do Brasil

Neste artigo propomos uma análise do trabalho doméstico não remunerado a partir da conjunção de duas perspectivas: a da estratificação social e a da economia doméstica. Para tal desenvolvemos uma escala de estratificação residencial, construída a partir do emprego da teoria da resposta ao item para a coleção dos bens empregados na produção doméstica em cada domicílio participante da Pesquisa de Usos do Tempo de Belo Horizonte. A utilização dessa escala possibilita, por um lado, a valoração de atividades não remuneradas no âmbito doméstico e da ocupação de "dona de casa", e por outro, o estudo da divisão sexual do trabalho por estrato social. A solidez da escala é indicada pela correlação extremamente alta entre ela e uma escala idêntica, construída a partir de dados da Pesquisa de Orçamentos Familiares, assim como pela correlação relativamente alta com outros índices de estratificação baseadas na ocupação dos indivíduos. Os principais resultados indicam, de um lado, a persistência da divisão sexual do trabalho em todos os estratos socioeconômicos, e, de outro, uma baixa correlação negativa entre status e tempo de cuidados domésticos, exceto para os homens durante fins de semana, quando a relação se torna positiva.

Palavras-chave: trabalho doméstico; divisão sexual do trabalho; estratificação residencial; teoria da resposta ao item; usos do tempo

\section{ABSTRACT}

Contributions to an Analysis of Residential Stratification, the Valuation of Domestic Labor, and Time Use in Brazil

The following article provides an analysis of unpaid domestic labor according to the alignment of two perspectives: social stratification and the domestic economy. To do so, we have developed a residential stratification scale based on an employment of item response theory for collection of the goods used in domestic production for homes participating in the Belo Horizonte Time Use Survey. The use of this scale facilitates a valuation of unpaid activities in the domestic sphere and the occupation of "housewife", as well as a study of the gender division of labor according to social stratum. The reliability of the scale is demonstrated by its extremely high correlation with an identical scale developed according to data from the Family Budget Survey, as well as its relatively high correlation with other stratification indexes based on the occupations of the individuals surveyed. The main results reveal both the persistence of the gender division of labor across all socioeconomic strata, as well as the low negative correlation between status and time spent on domestic tasks, except among men during weekends, when the relationship is positive.

Keywords: domestic labor; gender division of labor; residential stratification; item response theory; time use 
Estratificação Residencial, Valoração do Trabalho Doméstico e Uso do Tempo

RÉSUMÉ

Contributions à l'Analyse de la Stratification Résidentielle, de l'Estimation du Travail Domestique et de l'Usage du Temps au Brésil

Dans cet article, nous proposons une analyse du travail domestique non rémunéré à partir de la conjonction de deux perspectives, celle de la stratification sociale et celle de l'économie domestique. Nous avons élaboré à cette fin une échelle de stratification résidentielle en nous basant sur la théorie de la réponse à l'item pour la compilation des biens employés dans la production domestique de chaque foyer participant de la Recherche sur les usages du temps à Belo Horizonte. L'utilisation de cette échelle a d'un côté rendu possible l'estimation des activités non rémunérées dans le cadre domestique et du travail de "femme au foyer", et de l'autre, l'analyse de la division sexuée du travail par strate sociale. La pertinence de cette échelle tient à la corrélation extrêmement élevée entre celle-ci et une autre, identique, construite à partir des données de la Recherche sur les budgets familiaux, ainsi $q u^{\prime}$ à la concordance relativement haute avec d'autres indices de stratification basés sur l'occupation des individus. Les principaux résultats indiquent d'un côté la persistance de la division sexuée du travail au sein de l'ensemble des strates socioéconomiques, et de l'autre, une faible corrélation négative entre statut et temps de travail domestique, excepté pour les hommes durant les weekends, lorsque cette relation devient positive.

Mots-clés: travail domestique; division sexuée du travail; stratification résidentielle; théorie de la réponse à l'item; usages du temps

\section{RESUMEN}

Estratificación Residencial, Valoración del Trabajo Doméstico y Uso del Tiempo: Contribuciones al Análisis del Caso Brasileño

En este artículo, proponemos un análisis del trabajo doméstico no remunerado a partir de la conjunción de dos perspectivas: la de la estratificación social y la de la economía doméstica. Para ello, hemos desarrollado una escala de estratificación residencial fundamentada en la teoría de la respuesta al ítem para la recopilación de los bienes empleados en la producción doméstica en cada uno de los domicilios que participaron en el Estudio sobre los Usos del Tiempo de Belo Horizonte. La utilización de esta escala permite, por un lado, identificar las actividades no remuneradas en el ámbito doméstico y de las labores del "ama de casa" y, por otro, estudiar la división sexual del trabajo por estratos sociales. La solidez de la escala viene indicada por la correlación extremadamente alta entre esta y una escala idéntica construida a partir de datos del Estudio de Presupuestos Familiares, además de por la correlación relativamente alta con otros índices de estratificación basados en la ocupación de las personas. Los principales resultados indican, por una parte, la persistencia de la división sexual del trabajo en todos los estratos socioeconómicos y, por otra, una baja correlación negativa entre estatus y tiempo destinado a las labores domésticas, excepto en el caso de los hombres durante el fin de semana, cuando la relación pasa a ser positiva.

Palabras clave: trabajo doméstico; división sexual del trabajo; estratificación residencial; teoría de la respuesta al ítem; usos del tiempo

DADOS - Revista de Ciências Sociais, Rio de Janeiro, vol. 60, n² 2, 2017 